\title{
Fatal Complications during Anaesthesia in Moebius Syndrome: A Case Report and Brief Discussion of Relevant Precautions and Preoperative Assessments
}

Rasmussen $\mathrm{LK}^{1 *}$, Rian $\mathrm{O}^{1}$, Korshoej $\mathrm{AR}^{3}$, Christensen $\mathrm{S}^{4}$

${ }^{1}$ Department of Anaesthesiology, Regional Hospital Horsens, Denmark.

${ }^{2}$ Department of Neurosurgery, Aarhus University Hospital, Denmark.

${ }^{3}$ Department of Anaesthesiology and Intensive Care, Aarhus University Hospital, Denmark.

\begin{abstract}
We present a case of a 23-year-old male with Moebius syndrome admitted with sigmoid volvulus. Moebius Syndrome is a very rare complex congenital disorder characterised by uni- or bilateral facial paralysis resulting in orofacial abnormalities that in some individuals make tracheal intubation very difficult. In the present case, complications during the anaesthetic procedure caused a fatal outcome for the patient, as difficulties intubating was followed by an unexpected circulatory collapse, which could not be counteracted in the current setting.

The case emphasises the importance of handling patients with Moebius Syndrome and other patients with a difficult airway with utmost caution during anaesthetic care, particularly in the emergent setting where other complications might be present. A thorough preanaesthetic evaluation with specific attention to airway management and circulatory status is mandatory and all measures must be taken to optimise patient safety.
\end{abstract}

Keywords: Moebius Syndrome; Anaesthesia; Complications; Difficult Airway; Preoperative Assessment.

\section{*Corresponding Author:}

Line Kirkegaard Rasmussen,

Department of Anaesthesiology, Regional Hospital Horsens, 8700 Horsens, Denmark.

Tel: 415-606-1961

E-mail: line.kirkegaard@gmail.com

Received: June 01, 2015

Accepted: June 26, 2015

Published: July 01, 2015

Citation: Rasmussen LK, Rian O, Korshoej AR, Christensen S (2015) Fatal Complications During Anaesthesia in Moebius Syndrome: A Case Report and Brief Discussion of Relevant Precautions and Preoperative Assessments. Int J Anesth Res. 3(6), 116-118. doi: http://dx.doi. org/10.19070/2332-2780-1500030

Copyright: Rasmussen $\mathbf{L K}^{\odot}$ 2015. This is an open-access article distributed under the terms of the Creative Commons Attribution License, which permits unrestricted use, distribution and reproduction in any medium, provided the original author and source are credited.

\section{Introduction}

We report a case of a 23-year-old male with Moebius syndrome and unexpected cardiac arrest during intravenous anaesthesia induction. Moebius syndrome is a rare complex congenital disorder affecting approximately 1 in 50.000 to 1 in 500.000 newborns. It is characterised by underdevelopment of the sixth and seventh cranial nerve resulting in uni- or bilateral facial paralysis and involvement of other cranial nerves is also commonly seen. Furthermore, Moebius Syndrome is associated with congenital heart disease, such as ventricular septal defects, patent ductus arteriosus, and dextrocardia. Developmental delay is seen in approximately $10-15 \%$ and a higher incidence of autism spectrum disorders has also been reported $[1,2]$.

As a consequence of the orofacial abnormalities airway management can be very difficult, however, only few reports on the anaesthetic implications exist [1-3]. A difficult airway is defined as the clinical situation in which a conventionally trained anaesthesiologist experiences difficulty with facemask ventilation of the upper airway, difficulty with tracheal intubation, or both [4]. The incidence of failed intubation is approximately 1 in 1-2000 cases in the elective setting and approximately 1 in 50-100 cases in the emergent setting [5].

To the best of our knowledge this is the first case report to describe a fatal outcome in relation to failed intubation in Moebius Syndrome. In light of the presented case, we will discuss relevant precautions and appropriate preoperative assessments necessary when handling difficult airways and patients with Moebius Syndrome.

\section{Case}

A 23-year-old male with known Moebius syndrome was admitted with abdominal pain, excessive vomiting and diarrhoea. He had a surgical history of uncomplicated sigmoid volvulus relieved by colonoscopy three years before admission and nine years earlier he had undergone a lower spine surgery due to scoliosis. The patient had no medical history and did not take any medication. 
Because of his syndrome he suffered from deformation of hands and feet, unilateral absent pectoralis major, microstomia with a short tongue and high palate in addition to the characteristic facial paralysis and lateral gaze palsy. Furthermore, the patient was diagnosed with autism and slight developmental delay. An echocardiogram obtained nine years earlier concluded that the patient had a structurally normal heart.

On examination, the abdomen was found to be largely distended and painful on palpation and sigmoidoscopy with desufflation was performed under sedation and bag mask ventilation. However, symptoms relapsed after 24 hours. The patient was now vomiting and had severe nausea. CT-scan revealed considerable dilation of the distal colon $(8.5 \mathrm{~cm})$ and another attempt of sigmoidoscopy desufflation in general anaesthesia was planned on vital indication (Figure 1). The patient had no shortness of breath, chest pain or discomfort. Preoperative heart rate and blood pressure was 124 beats per minute (bpm) and 117/85 $\mathrm{mmHg}$, respectively. Body temperature was 38.3 degrees Celsius and the white blood cell count (WBC) was $12.3 \times 10^{\circ} / \mathrm{L}$. The patient thereby fulfilled the criteria of systemic inflammatory response syndrome (SIRS) [6]. Due to the vital indication of surgery the patient was brought to the operating theatre despite being non-fasting. Laboratory results revealed lactic acidosis $(\mathrm{pH} 7.28$, lactate $7.2 \mathrm{mmol} / \mathrm{L}$, carbon dioxide $4.2 \mathrm{kPa}$ and hydrocarbonate 16.0), but normal P-Potassium and P-Sodium $(3.5 \mathrm{mmol} / \mathrm{L}$ and $141 \mathrm{mmol} / \mathrm{L}$ respectively). The patient received approximately $1 \mathrm{~L}$ of Lactated Ringer's solution i.v. immediately before and during anaesthesia. A preoperative electrocardiogram (ECG) indicated QT prolongation with a corrected QT interval of $479 \mathrm{~ms}$ as the only abnormal finding.

The patient had a Simplified Airway Risk Index (SARI) score of 7 based on the history of difficult intubation, reduced neck movement, mouth opening and thyromental distance, Mallampati class III assignment and the lack of ability to pronate [7]. Based on this high-risk evaluation and a considerable risk of aspiration awake

Figure 1. Abdominal CT-scan illustrating the distended colon (left) and the sigmoid volvolus (right, white arrow).

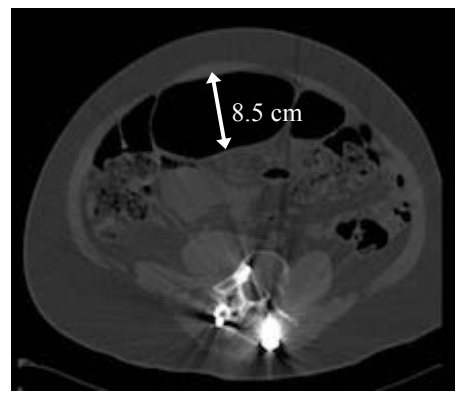

fiberoptic intubation was planned. The patient was monitored by a three lead ECG, non-invasive blood pressure measurement, and fingertip pulse oximetry. Attempts of airway topicalisation were made but failed due to cooperation difficulties. As a consequence, infusion of intravenous propofol and remifentanil $(10.4 \mu \mathrm{g} / \mathrm{kg} /$ min and $0.0875 \mu \mathrm{g} / \mathrm{kg} / \mathrm{min}$ respectively) was initiated in order to increase the level of sedation. However, the increased drug infusion resulted in prompt hypotension $(70 / 40 \mathrm{mmHg})$, which was immediately recovered to normal by administration of $20 \mathrm{mg}$ of Ephedrine. Blood oxygen saturation was kept in the normal range by uncomplicated bag mask ventilation. Continuous failure to cooperate was addressed by increasing remifentanil infusion to $0.125 \mu \mathrm{g} / \mathrm{kg} / \mathrm{min}$ resulting in a second hypotensive episode $(65 / 35 \mathrm{mmHg})$. Metaoxidrine $0.1 \mathrm{mg}$ was administered, where after the patient developed cardiac arrest. Cardiopulmonary resuscitation (CPR) was immediately initiated and the patient was treated according to the European Resuscitation (ERC) Guidelines. Four attempts of cardioversion were conducted along with the administration of both adrenaline and amiodarone. The saturation was never below 90\% during CPR although initially difficult to measure because of the poor perfusion. No reversible cause could be identified and therefore no specific treatment to correct such causes was given. Two further attempts of intubation by direct laryngoscopy and fiberoptic guidance were made under ongoing CPR but failed. Surgical cricothyroidotomy was not performed, as mask ventilation was uncomplicated. After 40 minutes the patient developed sustained asystolia and CPR was terminated.

\section{Discussion}

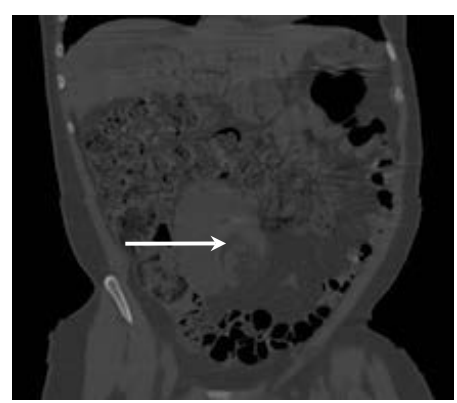

As described, management of the airways during anaesthesia can be difficult in patients with Moebius Syndrome. Indeed the incidence of difficult or failed intubation is considerably higher than normal. Ferguson (1996) [1] retrospectively reviewed the anaesthetic records of 19 children with Moebius Syndrome and found a high incidence of difficult $(42 \%)$ or failed $(10 \%)$ intubation. Similarly, Ames et al. [2] reviewed 111 paediatric anaesthesia records and encountered difficult tracheal intubation in $15.2 \%$ of patients although actual failure was rare (with endotracheal intubation performed in 106 cases). Despite well-characterised difficulties in managing the airways, this is the first report on a fatal outcome due to intravenous anaesthesia in a Moebius Syndrome patient.

In the presented case anaesthesia was required in order to counteract a life threatening surgical condition. The existing literature is mostly concerned with anaesthesia in relation to elective surgical procedures. It is likely that this difference could have influenced the outcome, as there are higher risks associated with anaesthesia in emergent conditions.

In concordance with what has previously been reported, management of the airways was extremely difficult in this case. Disease features such as micrognathia, microstomia, mandibular hypoplasia and cleft palate represents anatomical abnormalities that complicate airway management and predict difficult endotracheal intubation [2]. The patient presented with microstomia and a high palate, and a collective SARI score of $7(>3)$ indicating difficult direct laryngoscopy [7]. In connection with the sigmoidoscopy performed the day before, several attempts on unsuccessful intubation with both a video laryngoscope, intubating laryngeal mask 
and fiberoptic guidance had been made. The procedure was ultimately performed under sedation and bag mask ventilation and was uneventful. Based on guidelines for management of the difficult airway [4], the SARI-score and history of difficult intubation it was indeed anticipated that intubation would be difficult. In addition, a significant risk of aspiration was also present making sedation and bag mask ventilation an unsafe choice. Awake fiberoptic intubation therefore became the primary strategy for airway management and two senior anaesthesiologists both experienced with the technique were assigned to carry out the procedure. However, despite careful planning and several safety precautions the attempt of awake fiberoptic intubation failed. Considerable difficulties with patient cooperation undoubtedly contributed to this outcome and necessitated deeper sedation. Although, mask ventilation was uncomplicated at all times keeping the blood oxygen saturation above $92 \%$, the patient developed a surprising circulatory collapse in connection with increased dose of sedatives, despite the fact that the doses administered were well within the normal safety range. Furthermore, the circulatory collapse was not a result of desaturation.

Prior to the anaesthetic care blood pressure had been stabile and within the normal range. Tachycardia (110-120 bpm) was present but also stabile and interpreted as the result of a painful condition and mild sepsis in combination. The patient had not previously shown any signs of abnormally high sensitivity to anaesthetic drugs. The hemodynamic instability that followed anaesthesia induction with low doses of propofol and remifentanil was therefore highly unexpected. The patient had a metabolic acidosis with elevated lactate and at the same time also fulfilled the criteria of sepsis. Elevated lactate is commonly seen in acutely ill patients and may be associated with a variety of causal relations [8]. In the current context, we do not consider the elevated levels of lactate a sole result of sepsis since there was nothing to indicate severe sepsis or septic shock at the time. Furthermore, the relatively low CRP of $57 \mathrm{~g} / \mathrm{L}$ questions the importance of infection in this context. Mesenteric ischemia is also associated with elevated levels of lactate and given the considerable dilation of the distal colon this could indeed also have contributed to the observed lactate elevation. A combination of sepsis, possible dehydration caused by the abdominal symptoms and some degree of mesenteric ischemia most likely represents the reasons for the metabolic acidosis observed.

Despite difficulties intubating, the presence of metabolic acidosis and mild sepsis, which both represent fairly common challenges to anaesthesiologists, we consider the presented course of events unexpected and illustrative of the overall complexity of this and other Moebius Syndrome cases. The initial primary focus was safe management of the patient's high-risk airways, as the circulatory status was considered near normal and stabile upon clinical assessment. However, no echocardiographic examination was performed prior to surgery, which clearly represents a limitation to the care of this patient. The sum of challenges, the sum of challenges ranging from cooperation to intubation difficulties led to an unexpected circulatory collapse, which could not be counteracted in the given setting. Although compromised hemodynamics were not anticipated, further precautions such as optimised patient monitoring (arterial pressure), placement of a central venous catheter for pharmacological cardiac support (including fluid therapy) and alternative choices of medication for anaesthesia (small doses of ketamine) could perhaps have increased the chances of a favourable outcome. This case highlights the importance of aggressive preoperative fluid therapy guided by both close hemodynamic assessment and frequent measurement of electrolytes and $\mathrm{pH}$, and emphasises the importance of thorough cardiac assessment in complicated anaesthetic cases.

\section{Conclusion}

The presented case illustrates an extremely challenging case of anaesthesia and the severity of anaesthetic complications that can occur when handling Moebius Syndrome patients. The thoughts and considerations derived from our experience apply to any critically ill patient with a difficult airway. In fact, it is our belief that the fatal outcome of this case was unrelated to the patient's syndrome, but rather related to the combination of acute illness, difficult airway, and cooperation difficulties.

We find that thorough preanaesthetic evaluation is of utmost importance when conducting acute anaesthetic procedures on patients with Moebius Syndrome, or on any patient where difficult intubation is expected, in order to prevent serious complications. Furthermore, we find that the assessment of airways should also include identification of individual disease features including. expected level of cooperation in addition to standard multivariate risk predictors of difficult tracheal intubation. In order to ensure the highest level of caution we recommend that these patients be handled by senior anaesthesiologists and that all measures are taken to ensure proper circulatory control. Specifically, we recommend that careful attention is directed towards correction of underlying water and electrolytic abnormality before anaesthesia induction and furthermore that a preoperative circulatory assessment should include ECG and echocardiography as mandatory elements in order to evaluate immediate myocardial performance and hemodynamic status. Such precautions will expectedly decrease the risk of unexpected fatalities and complications in difficult cases such as Moebius Syndrome.

\section{References}

[1]. Ferguson S (1996) Moebius syndrome: a review of the anaesthetic implications. Paediatr Anaesth 6(1): 51-56.

[2]. Ames W, Shichor T, Speakman M, Zuker R, McCaul C (2005) Anesthetic management of children with Moebius sequence. Can J Anesth 52(8): 837844.

[3]. Gondipalli P, Tobias JD (2006) Anesthetic implications of Möbius syndrome. J Clin Anesth 18(1): 55-59.

[4]. Apfelbaum JL, Haberg CA, Caplan RA, Blitt CD, Connis RT, et al. (2013) Practice guidelines for management of the difficult airway: an updated report by the American Society of Anesthesiologists Task Force on Management of the Difficult Airway. Anesthesiology 118(2): 251-270.

[5]. Cook T, MacDougall-Davis SR (2012) Complications and failure of airway management. Br J Anaest 109(suppl 1): i68-i85.

[6]. Levy M, Fink M, Marshall J, Abraham E, Angus D, et al. (2003) 2001 SCCM/ESCIM/ACCP/ATS/SIS International Sepsis Definitions Conference. Crit Care Med 31(4): 1250-1256

[7]. el-Ganzouri AR, McCarthy RJ, Tuman KJ, Tanck EN, Ivankovich AD (1996) Preoperative airway assessment: predictive value of a multivariate risk index. Anesth Analg 82(6): 1197-1204.

[8]. Andersen L, Mackenhauer J, Roberts J, Berg K, Cocchi M, et al. (2013) Etiology and therapeutic approach of elevated lactate. Mayo Clin Proc 88(10): $1127-1140$. 